\title{
Letter
}

\section{Saccharomyces boulardii fungaemia in an intensive care unit patient treated with caspofungin}

\author{
Nikolaos Lolis ${ }^{1}$, Dimitrios Veldekis ${ }^{1}$, Hellen Moraitou ${ }^{2}$, Sofia Kanavaki ${ }^{2}$, Aristea Velegraki ${ }^{4}$, \\ Charis Triandafyllidis ${ }^{1}$, Chronis Tasioudis ${ }^{1}$, Angellos Pefanis ${ }^{5}$ and loannis Pneumatikos ${ }^{3}$
}

\begin{abstract}
'Sotiria General Hospital Respiratory Intensive Care Unit, 'Sotiria' General Hospital of Athens, Mesogion 152, 11527 Athens, Greece 2Sotiria General Hospital Microbiology Laboratory, 'Sotiria' General Hospital of Athens, Mesogion 152, 11527 Athens, Greece

${ }^{3}$ Critical Care Unit, University Hospital of Alexandroupolis, Dragana 1, 68100 Alexandroupolis, Greece

${ }_{4}^{4}$ Mycology Reference Laboratory (Hellenic Centre for Diseases Control), Laiko Hospital, Goudi 11526, Athens, Greece

$53^{\text {rd }}$ Department of Internal Medicine, 'Sotiria' General Hospital of Athens, Mesogion 152, 11527 Athens, Greece
\end{abstract}

Corresponding author: loannis Pneumatikos, ipnevmat@med.duth.gr

Published: 9 April 2008

This article is online at http://ccforum.com/content/12/2/414

(c) 2008 BioMed Central Ltd
Critical Care 2008, 12:414 (doi:10.1186/cc6843)

on the DNA of the strain isolated as $S$. cerevisiae, as well as the isolate of $S$. boulardii obtained from the commercially available product, revealed $98 \%$ correspondence, which is considered almost absolute identity. Given the microbiological finding that $S$. cerevisiae is undistinguishable from S. boulardii, we believe that fungaemia was a consequence of treatment with this yeast [4].

Saccharomyces boulardii is frequently used in critically ill patients to treat diarrhoea, and it is the only yeast probiotic that has been proved to be effective in double-blind studies $[1,2]$. Although it is considered a safe biotherapeutic agent, the incidence of fungaemia has increased in recent years [3].

A 56-year-old male patient was transferred to our intensive care unit with pneumonia and septic shock. Five days previously he had been intubated and admitted to the coronary care unit because of acute pulmonary oedema. Despite antibiotic treatment and supportive therapy in the intensive care unit, fever continued and both Acinetobacter baumannii and Klebsiella pneumoniae were isolated from bronchial secretions. Catheter-tip culture of the central venous catheter remained sterile. The patient then developed major diarrhoea (four to eight liquid stools per day). His serum was negative for Clostridium difficile toxin, and stool culture did not reveal any pathogens. Treatment with Ultra Levure (S. boulardii, Biocodex, Beauvais, France) $500 \mathrm{mg}$ four times daily via feeding tube was started.

One week later, two blood cultures were found to be positive. A saccharomyces strain was identified as Saccharomyces cerevisiae. Susceptibility testing indicated the presence of a strain susceptible to caspofungin, treatment with which was initiated. The patient then improved and four subsequent blood cultures were found to be sterile. Sequencing analysis
Three hypotheses have been reported for the pathogenesis of $S$. boulardii fungaemia [2]: yeast translocation across the gut mucosa, contamination of central venous catheter and massive colonization. Our patient's fungaemia was probably the result of translocation of $S$. boulardii as a consequence of septic shock and intestinal ischaemia.

S. boulardii fungaemia is usually treated with amphotericin B or fluconazole. We admininstered caspofungin with excellent results. This is the first case in the literature of $S$. boulardii being treated with caspofungin.

We conclude that the incidence of $S$. boulardii fungaemia is probably underestimated in critically ill patients. When a patient is treated with $S$. boulardii, health care professionals must wear gloves when they open the drug packaging and must do so outside the patient's room. The potential therapeutic benefit of $S$. boulardii should carefully be evaluated in patients with septic shock. If contamination of vascular catheters is suspected, then removal of the central catheter should be considered.

\section{Competing interests}

The authors declare that they have no competing interests. 


\section{References}

1. Czeruckat D, Piche P. Rampal P: Review article: yeast as probiotics - Saccharomyces boulardii. Aliment Pharmacol Ther 2007, 26:767-778.

2. Bleichner G, Blehaut H, Mentec H, Moyse D: Saccharomyces boulardii prevents diarrhea in critically ill tube-fed patients. A multicenter, randomized, double-blind placebo-controlled trial. Intensive Care Med 1997, 23:517-523.

3. Lherm T, Monet C, Nougière B, Soulier M, Larbi D, Le Gall C, Caen D, Malbrunot C: Seven cases of fungemia with Saccharomyces boulardii in critically ill patients. Intensive Care Med 2002, 28:797-801.

4. Basseti S, Frei R, Zimmerli W: Fungemia with Saccharomyces cerevisiae after treatment with Saccharomyces boulardii. Am J Med 1998, 105:71-72. 